\title{
Chronic Lymphocytic Leukemia (CLL)/Small Lymphocytic Lymphoma (SLL) - all the aspects
}

\author{
Authors: \\ Azra Jahic, $\mathrm{MD}, \mathrm{PhD}^{1}$, Samira Hasic Custendil, $\mathrm{MD}, \mathrm{PhD}^{1}$ \\ 1 \\ Hematology Department, Clinic for Oncology, Hematology and Radiotherapy, University \\ Clinical Center Tuzla, Tuzla, 75 000, Bosnia and Herzegovina
}

\section{Corresponding author:}

Azra Jahic, MD, PhD, Hematology Department, Clinic for Oncology, Hematology and Radiotherapy, University Clinical Center Tuzla, Tuzla, 75 000, Bosnia and Herzegovina, E-mail address: azrasong1@yahoo.com

\begin{abstract}
B-chronic lymphocytic leukemia (B-CLL)/Small lymphocytic lymphoma (SLL) is a neoplastic, lymphoproliferative disease, characterized by accumulation of small, mature lymphocytes of the B- cell line in the blood, bone marrow and lymphoid tissues. CLL and SLL are different manifestations of the same disease. The major difference is that in CLL a significant number of the abnormal lymphocytes are also found in the bone marrow and blood, while in SLL the abnormal lymphocytes are predominantly found in the lymph nodes and bone marrow. B-CLL is the commonest leukaemia in adults in the Western countries. The mean age of occurrence is in the range of 64-70 years. Adequate immunophenotyping of peripheral blood is essential for establishing the diagnosis of CLL/SLL. The typical immunophenotype for CLL/SLL is CD5+, CD10-, CD19+, and CD20 dim, surface immunoglobulin dim, CD23+, CD43 +/-, and cyclin D1-. The clinical course of the disease is highly variable. Approximately $2-10 \%$ of patients with CLL/SLL will develop histologic transformation to diffuse large B-cell lymphoma or Hodgkin lymphoma. This transformation is called Richter's syndrome and involves a much more aggressive disease and a fatal outcome. During the past decade, numerous prognostic factors were identified and include serum markers such as thymidine kinase and beta- 2 microglobulin, genetic markers including IGHV mutational status and cytogenetic abnormalities detected by FISH (e.g., del(13q), del(11q), del(17p), CD38 expression, CD49d and ZAP-70 expression/methylation). During the last few years, recurrent mutations in NOTCH1, SF3B1 and BIRC3 genes with prognostic implications in CLL were also identified. Minimal residual disease (MRD) negativity determined in the peripheral blood after the end of treatment is emerging as an important predictor of treatment efficacy. Ongoing preclinical and clinical studies will unravel newer therapeutic targets and keep on improving patient outcomes and the quality of life.
\end{abstract}

Key words: B-chronic lymphocytic leukemia (B-CLL)/Small lymphocytic lymphoma (SLL), immunophenotype, prognostic factors. 


\section{Introduction}

B-chronic lymphocytic leukemia (BCLL)/Small lymphocytic lymphoma (SLL) is a neoplastic, lymphoproliferative disease, characterized by accumulation of small, mature lymphocytes of the B-cell line in the blood, bone marrow and lymphoid tissues. CLL and SLL are different manifestations of the same disease, and are managed in much the same way.

(1). The major difference is that in CLL a significant number of the abnormal lymphocytes are also found in the bone marrow and blood, while in SLL the abnormal lymphocytes are predominantly found in the lymph nodes and bone marrow. In the case of bone marrow cells affected by SLL, the pattern of infiltration is usually nodular rather than diffuse or interstitial.

(2). According to the World Health Organization, CLL and SLL represent one disease at different stages, not two separate entities (3). In less than $2 \%$ of cases, neoplastic lymphocytes are the origin of $\mathrm{T}$-cell line when they are considered $\mathrm{T}$-cell prolymphocytic leukemia. Diagnosis of B-CLL requires monoclonal B-lymphocytosis greater than $5000 / \mu 1$ $\left(5 \times 10^{9} / 1\right)$ which is established by flow cytometry quantification (4). The diagnosis of SLL requires the presence of lymphadenopathy and/or splenomegaly with less than $5000 \mathrm{~B}$-lymphocytes/ $\mu 1\left(5 \times 10^{9} / \mathrm{L}\right)$ in the peripheral blood (4). The presence of fewer B-cells in the absence of palpable lymphadenopathy, or other clinical features characteristic of a lymphoproliferative disorder, is defined as monoclonal Blymphocytosis (MBL). The diagnosis of MBL includes absolute B-lymphocyte count of less than $5000 / \mathrm{mm}^{3}$, lymph nodes less than $1.5 \mathrm{~cm}$, no thrombocytopenia or anemia. MBL is a relatively recent diagnostic category describing individuals with an abnormal B-cell population of immunophenotype of CLL, but do not meet diagnostic criteria for CLL (5). Morphologically, the leukemic cells generally appear similar to normal resting lymphocytes. Typically, these cells have scanty, bluish cytoplasm upon WrightGiemsa staining, moderately condensed and mature-appearing nuclei (Figure 1). A few cells can have prominent nucleoli. During the preparation of the blood film, many CLL lymphocytes are noticeably disrupted and appear as smudge cells. Leukemic leucocytosis in excess of $800,000 / \mu 1$ $\left(800 \times 10^{9} / 1\right)$ may produce blood hyperviscosity. 


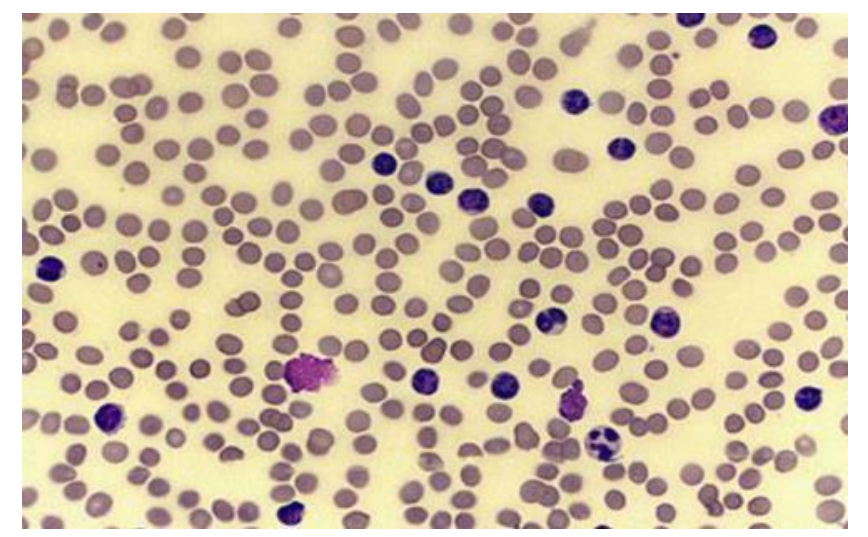

Figure 1. Peripheral smear from a patient with chronic lymphocytic leukemia, small lymphocytic variety.

\section{Etiology and pathogenesis}

Physiological maturation of B-cells include numerous processes of modification such as editing of B-cell receptors, the inclusion of an isotype or class, somatic hypermutation, the gene rearrangement of light, and heavy chains of immunoglobulins. All these processes include DNA damage and repair. Within the framework of proliferation of B-cells, possible errors that might occur during DNA repair are associated with the genesis of B-lymphoid neoplasms $(6,7)$. Unlike other leukemia, CLL is not associated with exposure to ionizing radiation, sunlight, pesticides or known carcinogens $(8,9)$. Although most cases of CLL occur sporadically, many cases of this disease can be found within a family. What is more, some authors consider hereditary factors important when it comes to the development of this disease (10). The first degree relatives of patients with CLL have a three times greater risk of developing the disease compared to the general population (11). Patients within these families are younger than patients with sporadic CLL, suggesting that genetic factors in familial CLL affect early leukemogenesis (10).

\section{Incidence}

B-chronic lymphocytic leukaemia is the most common leukaemia in adults in the Western countries, but rare in East Asia and Japan. Since the disease is relatively indolent, CLL accounts $0.8 \%$ of all cancers, $30 \%$ of leukemias and approximately $7 \%$ of newly diagnosed cases of NHL (12). In Japan, for instance, CLL accounts for less than $6 \%$ of all leukemia (13). However, the incidence of CLL in Korea amounted to only $1.5 \%$ rate in the US (14). The incidence of this disease in Israel is significantly higher among European immigrants compared to immigrants from Africa and Asia (15). The incidence in men is twice more common than in women (16). The risk of developing CLL increases progressively with age. The incidence of this disease is 2.8 times higher for older men than for 
older women (17). At the time of diagnosis, most patients are older than 60 years, and $90 \%$ are older than 50 years. The mean age of occurrence is in the range of 64-70 years (18), and rarely occurs in people under the age of 25 years.

\section{Clinical features}

Approximately $70 \%$ of CLL patients have no symptoms at the time of diagnosis. Symptoms such as reduced exercise tolerance, fatigue or malaise, may be present even in the absence of anemia and hepatosplenomegaly. In advanced stages of the disease, unexplained weight loss, recurring infections, and bleeding may be present due to thrombocytopenia or symptoms of anemia. Often, night sweats or low grade fevers can be present as the so-called B-symptoms. Approximately half of all CLL patients present with mild to moderate splenomegaly, which occasionally can give a feeling of fullness or pressure in the abdomen. Patients with CLL may develop anemia secondary to leukemic marrow infiltration, the myelosupressive effect of chemotherapy and inhibiting cytokines, autoimmunity directed against red cell antigens, hypersplenism and/or a poor nutritional status that leads to deficiency of folic acid, vitamin B12, or iron. At any stage, patients can develop immune thrombocytopaenia because of antiplatelet antibodies. Less frequently, patients develop hepatomegaly secondary to leukemic cell infiltration of the liver.
Nearly $80 \%$ of patients have painless enlargement of lymph glands, predominantly cervical, supraclavicular or axillary region. Lymphadenopathy rarely leads to obstruction of vascular or lymphatic channels, but represent a viable reason to take into consideration the possibility of a secondary pulmonary neoplasm in the case of superior vena cava syndrome. Massive mesenteric or abdominal lymphadenopathy can rarely cause lower extremity edema, secondary to compression of the inferior vena cava or ureteral obstruction and hydronephrosis. Obstruction of the biliary tract caused by periportal lymphadenopathy is rare. Organ infiltration with leukemic cells is frequently detected at autopsy. Impaired function of these organs occurs only if leukemic cells infiltrate special places, such as the retro-orbit, scalp, subconjunctivae, prostate, gonads, pharynx, pericardium, lung tissue, pleura or the lining of the gastrointestinal system (19). Leukemic cell infiltration of the central nervous system (CNS) is unusual, but may produce headache, meningitis, cranial nerve palsie, or coma (20). Less common symptom includes chronic rhinitis when leukemic cells compromise the nasal cavity $(18,21)$. Some patients present recurrent infections or autoimmune hemolytic anemia a few months before the diagnosis of CLL. The most commonly found pathogens are Streptococcus pneumoniae, Staphylococcus, Haemophilus influenzae 
and Herpes virus. Patients with CLL are prone to viral or bacterial infections as a result of impaired T-cell immunity or hypogammaglobulinemia. The clinical course of the disease is completely heterogeneous. Approximately 2-8\% of patients with CLL/SLL will develop histologic transformation to diffuse large B-cell lymphoma during the course of their disease and treatment (22). This transformation is called Richter's syndrome and the mean time of its occurrence from the time of diagnosis of CLL is two years (23). Richter's syndrome involves a much more aggressive disease and a fatal outcome. Large B-cell lymphoma that arises from the context of the disease may be preceded by CLL (24). In some cases, the Epstein-Barr virus infection and immunosuppressive treatment of CLL promote this transformation (24). Less than $1 \%$ of patients develop classical Hodgkin lymphoma (22), which usually occurs in patients with the mutation of $\mathrm{IgVH}$ gene and unrelated to CLL clone. Patients with CLL have an increased risk for the development of secondary malignancies such as sarcoma, melanoma, carcinoma of the larynx, lung, urinary bladder and stomach, without any causal relationship between pretreatment and secondary neoplasms (25).

\section{Diagnosis and prognosis}

Adequate immunophenotyping of peripheral blood is essential for establishing the diagnosis of CLL/SLL. The typical immunophenotype for CLL/SLL is CD5+, CD10-, CD19+, and CD20 dim, surface immunoglobulin dim, CD23+, CD43 +/-, and cyclin D1-. Bone marrow biopsy is generally not required for the diagnosis. Absence of cyclin D1 expression is critical in differentiation of CLL/SLL from Mantle Cell Lymphoma (MCL), as they are both CD5+ B-cell tumors. FISH analysis for $\mathrm{t}(11 ; 14)$ can also help to distinguish MCL from CLL. FISH for the detection of $\operatorname{del}(11 \mathrm{q})$, $\operatorname{del}(13 q)$, trisomy $12, \operatorname{del}(17 p)$, stimulated metaphase karyotype and molecular genetic analysis (by PCR or sequencing) to detect IGHV mutation status and TP53 mutations can provide useful prognostic information and may guide selection of therapy. Conventional metaphase cytogenetics is difficult in CLL as a result of the very low in vitro proliferative activity of the leukemic cells. Therefore, interphase cytogenetic analysis with FISH is the standard method to detect chromosomal abnormalities that may have prognostic significance. However, FISH can only detect abnormalities specific to the probes utilized. Cytokine or $\mathrm{CpG}$ oligonucleotide stimulation was utilized to enhance metaphase analysis (26). Recent reports suggest that complex karyotype ( $\geq 3$ unrelated chromosomal abnormalities in more than one cell on conventional karyotyping of stimulated CLL cells) is associated with an unfavorable prognosis (27-29). In one report, complex karyotype was significantly associated with 
unmutated IGHV and aberrations of chromosome $17 \mathrm{p}$, and it was also identified as an independent prognostic factor for shorter time-to-first-treatment (27). In patients with relapsed or refractory CLL treated with ibrutinibbased regimens, complex karyotype was associated with disease progression, inferior event free survival (EFS) and overall survival (OS) $(28,29)$. Cytogenetic abnormalities can evolve over time; therefore, re-evaluation of FISH and karyotype is necessary to direct treatment options in patients with indications for treatment. Histological analysis can objectify four patterns of bone marrow infiltration by malignant B-lymphocytes: nodular, interstitial, diffuse and mixed pattern nodular-interstitial infiltration (30). Approximately $1 / 3$ of the patients have an interstitial pattern of bone marrow infiltration which is associated with better prognosis and/or with early stage of the disease. Approximately $10 \%$ of patients have a nodular pattern of bone marrow infiltration and approximately $25 \%$ of patients have a mixed pattern nodularinterstitial. In $1 / 4$ of patients, the bone marrow infiltration pattern can be found, which is related to the advanced clinical stage and/or with more aggressive disease (31). Though classically, the pattern of bone marrow involvement (diffuse vs. nodular) had prognostic significance, this is no longer a factor when one uses more reliable prognostic markers such as IGHV mutational status and cytogenetic abnormalities determined by FISH, all of which can be obtained by analysis of circulating lymphocytes. Thus, bone marrow biopsy is no longer considered a required part of the diagnostic evaluation of patients with suspected CLL, though it remains useful to evaluate the etiology of cytopenias.

\section{Staging}

The nearly universal involvement of the bone marrow and peripheral blood in CLL/SLL limits the utility of the Ann Arbor staging system. Two staging systems, the Rai and Binet systems are currently used worldwide in the evaluation of patients with CLL (32,33). The modified Rai classification stratifies patients into 3 risk groups (Figure 2) (32). Survival of patients with low-risk disease (Rai stage 0; median survival 150 months) is essentially the same as the survival rate of age-matched controls. Patients with intermediate-risk disease (Rai stage I-II; median survival 71-101 months) have shorter survival, particularly when other adverse factors coexist, such as a lymphocyte doubling time of less than one year. Patients with high-risk features (Rai stage III-IV; median survival 19 months) have poor prognosis (Figure 2) (32). The Binet staging system is based on the number of involved areas and the level of hemoglobin and platelets and similar to the Rai staging system, provides meaningful correlation with clinical outcome (Figure 3) (33). 
Medical Research Archives, Vol. 5, Issue 3, March 2017

Chronic Lymphocytic Leukemia (CLL)/Small Lymphocytic Lymphoma (SLL) - all the aspects

\begin{tabular}{|l|l|l|}
\hline Stage & Description & Risk status \\
\hline 0 & $\begin{array}{l}\text { Lymphocytosis, lymphocytes in blood }>15,000 / \mu 1 \\
\text { and }>40 \% \text { lymphocytes in the bone marrow }\end{array}$ & Low \\
\hline I & Stage 0 with enlarged node(s) & Intermediate \\
\hline II & Stage 0-I with splenomegaly, hepatomegaly, or both & Intermediate \\
\hline III & $\begin{array}{l}\text { Stage 0-II with hemoglobin }<11,0 \mathrm{~g} / \mathrm{dl} \text { or hematocrit } \\
<33 \%\end{array}$ & High \\
\hline IV & Stage 0-III with platelets $<100,000 / \mu 1$ & High \\
\hline
\end{tabular}

Figure 2. Rai System. *Immune-mediated cytopenias are not the basis for these stage definitions.

\begin{tabular}{|l|l|}
\hline Stage & Description \\
\hline A & $\begin{array}{l}\text { Hemoglobin } \geq 10 \mathrm{~g} / \mathrm{dL} \text { and } \\
\text { Platelets } \geq 100,000 / \mathrm{mm}^{3} \text { and } \\
<3 \text { enlarged areas }\end{array}$ \\
\hline B & $\begin{array}{l}\text { Hemoglobin } \geq 10 \mathrm{~g} / \mathrm{dL} \text { and } \\
\text { Platelets } \geq 100,000 / \mathrm{mm}^{3} \text { and } \\
\geq 3 \text { enlarged areas }\end{array}$ \\
\hline C $^{*}$ & $\begin{array}{l}\text { Hemoglobin }<10 \mathrm{~g} / \mathrm{dL} \text { and/or } \\
\text { Platelets }<100,000 / \mathrm{mm}^{3} \text { and } \\
\text { any number of enlarged areas }\end{array}$ \\
\hline
\end{tabular}

Figure 3. Binet System. ${ }^{*}$ Immune-mediated cytopenias are not the basis for these stage definitions.

\section{Prognostic Factors}

During the past decade, numerous factors were identified and include serum markers such as thymidine kinase and beta-2 microglobulin, genetic markers including IGHV mutational status and cytogenetic abnormalities detected by FISH (e.g., del(13q), del(11q), del(17p), CD38 expression, CD49d and ZAP-70 expression/methylation). Unmutated IGHV or the use of VH3-21 was shown to be independent predictors of shorter treatment-free interval and/or survival outcomes, even when there are high-risk genomic abnormalities (34-37). Expression of CD38 $(\geq 7 \%$ of $\mathrm{B}$ lymphocytes) (35,36,38-41) and/or ZAP70 ( $\geq 20 \%$ of B lymphocytes) (42-44) were 
also associated with shorter progressionfree survival (PFS) and OS outcomes. Elevated level of serum beta- 2 microglobulin was shown to be a strong independent prognostic indicator for treatment-free interval, response to treatment, and OS, including in patients treated with first-line chemoimmunotherapy regimens (45-47). One of the advantages of beta- 2 microglobulin is that it is readily measured by standard laboratory evaluation of blood samples. However, it is influenced in a CLL disease independent manner by renal dysfunction.

Cytogenetic abnormalities that can be detected by FISH are present in over $80 \%$ of patients with previously untreated CLL. The most common abnormality is $\operatorname{del}(13 q)(55 \%)$ as a sole finding, followed by $\operatorname{del}(11 q)(18 \%)$, trisomy $12(16 \%)$, $\operatorname{del}(17 p)(7 \%)$, and $\operatorname{del}(6 q)(7 \%)(48)$. $\operatorname{Del}(13 q)$ as a sole abnormality is associated with favorable prognosis and the longest median survival (133 months). $\operatorname{Del}(11 \mathrm{q})$ is often associated with extensive lymphadenopathy, disease progression and shorter median survival (79 months) (48). Among patients with del(11q), those with a complete loss of ATM function might have impaired response to irradiation or cytotoxic drugs, resulting in poor clinical outcome (49). Recent studies showed that previously untreated patients with del(11q) respond well to combination therapy with fludarabine and cyclophosphamide (FC), suggesting that the addition of an alkylating agent to fludarabine may help to overcome the adverse prognostic significance of del(11q) in patients with CLL (36,50). Del(17p), which reflects the loss of the TP53 gene and is frequently associated with mutations in the remaining TP53 allele, is associated with worst outcomes, with short treatment-free interval, short median survival (32 months), and poor response to chemotherapy (48). Abnormalities of TP53 can be observed in the absence of $\operatorname{del}(17 \mathrm{p}) \quad(51,52)$. Studies with fludarabine-based regimens identified TP53 mutations as an independent predictor of decreased survival and resistance to chemotherapy (51-53). During the last few years, recurrent mutations in NOTCH1, SF3B1 and BIRC3 genes with prognostic implications in CLL were identified (54-58). Data from prospective clinical trials confirmed that NOTCH1 and SF3B1 mutations are predictors of shorter survival in patients with newly diagnosed as well as relapsed or refractory CLL (52,59). NOTCH1 mutation was also independently associated with Richter's transformation $(60,61)$. In the general clinical practice setting, prognostic factors should not determine treatment choices, with the exception of del(17p) or del(11q).

\section{Minimal residual disease (MRD)}

Minimal residual disease (MRD) negativity determined in the peripheral 
Chronic Lymphocytic Leukemia (CLL)/Small Lymphocytic Lymphoma (SLL) - all the aspects

blood after the end of treatment is emerging as an important predictor of treatment efficacy. In the combined analysis of two phase III GCLLSG studies, among patients who achieved CR, there was a statistically significant difference in PFS between MRD-negative and MRD-positive patients (69.2 months vs. 40.4 months; $\mathrm{P}=.001$ ) (62). The persistence of post treatment splenomegaly as a sole abnormality in MRD-negative patients had no negative influence on PFS. These results support the use of MRD for response evaluation.

\section{Summary}

Our understanding of chronic lymphocytic leukemia has undergone a dramatic change and is constantly evolving. Defining prognostic parameters potentiating division of patients in groups with favorable and unfavorable prognosis could help the benefit assessment of early treatment, improve treatment effects, and potentiate treatment modification for each patient. Ongoing preclinical and clinical studies will unravel newer therapeutic targets and keep on improving patient outcomes and the quality of life. 
Chronic Lymphocytic Leukemia (CLL)/Small Lymphocytic Lymphoma (SLL) - all the aspects

\section{References:}

1. Tsimberidou AM, Wen S, O'Brien $\mathrm{S}$, et al. Assessment of chronic lymphocytic leukemia and small lymphocytic lymphoma by absolute lymphocyte counts in 2,126 patients: 20 years of experience at the University of Texas M.D. Anderson Cancer Center. J. Clin. Oncol. 25, 46484656 (2007).

2. Kipps TJ, Robbins BA, Tefferi A, et al. CD5-positive B-cell malignancies frequently express cross-reactive idiotypes associated with IgM autoantibodies. Am. J. Pathol. 136, 809-816 (1990).

3. Simonsson B, Wibell L, Nilsson $\mathrm{K}$, Beta 2-microglobulin in chronic lymphocytic leukaemia. Scand. J. Haematol. 24, 174-180 (1980).

4. Hallek M, Cheson BD, Catovsky $\mathrm{D}$, et al. Guidelines for the diagnosis and treatment of chronic lymphocytic leukemia: a report from the International Workshop on Chronic Lymphocytic Leukemia updating the National Cancer Institute-Working Group 1996 guidelines. Blood 111, 54465456 (2008).

5. Rawstron AC, Monoclonal B-cell lymphocytosis. Hematology Am. Soc. Hematol. Educ. Program. 430-439 (2009).
6. Kuppers R, Klein U, Hansmann ML, Rajewsky K, Cellular origin of human B-cell lymphomas. $N$. Engl. J. Med. 341(20), 1520-1529 (1999).

7. Kuppers, R, Mechanisms of B-cell lymphoma pathogenesis. Nat. Rev. Cancer. 5(4), 251-262 (2005).

8. Rushton L, Romaniuk H, A casecontrol study to investigate the risk of leukaemia associated with exposure to benzene in petroleum marketing and distribution workers in the United Kingdom. Occup. Environ. Med. 54, 152-166 (1997).

9. Inskip PD, Kleinerman RA, Stovall M, et al. Leukemia, lymphoma and multiple myeloma after pelvic radiotherapy for benign disease. Radiat. Res. 135, 108-125 (1993).

10. Yuille MR, Matutes E, Marossy A, et al. Familial chronic lymphocytic leukemia: A survey and review on of published studies. $B r . J$. Haematol. 109(4), 794-799 (2000).

11. Cuttner J, Increased incidence of hematologic malignancies in firstdegree relatives of patients with chronic lymphocytic leukemia. Cancer Invest. 10, 103-109 (1992).

12. A clinical evaluation of the International Lymphoma Study Group classification of non- 
Chronic Lymphocytic Leukemia (CLL)/Small Lymphocytic Lymphoma (SLL) - all the aspects

Hodgkin's lymphoma. The NonHodgkin's Lymphoma Classification Project. Blood 89, 3909-3918 (1997).

13. Tamura K, Sawada H, Izumi Y, et al. Chronic lymphocytic leukaemia (CLL) is rare, but proportion of $\mathrm{T}$ CLL is high in Japan. Eur. J. Haematol. 67, 152-157 (2001).

14. Ahn Jo, Koo HH, Park BJ, et al. Incidence estimation of leukemia among Koreans. J. Korean. Med. Sci. 6, 299-307 (1991).

15. Bartal A, Bentwich Z, Manny N, Izak G, Ethnical and clinical aspects of chronic lymphocytic laeukemia in Israel: A survey on 288 patients. Acta Haematol. 60,161-171 (1978).

16. Cartwright RA, Gurney KA, Moorman AV, Sex ratios and the risks of haemathologic malignancies. Br. J. Haematol. 118, 1071-1077 (2002).

17. Diehl LF, Karnell LH, Menck HR, The American College of Surgeons Commision on Cancer and the American Cancer Society. The National Cancer Data Base report on age, gender, treatment, and outcomes of patients with chronic lymphocytic leukemia. Cancer 86, 2684 (1999).

18. Redaelli A, Laskin BL, Stephens $\mathrm{JM}$, et al. The clinical and epidemiological burden of chronic lymphocytic leukaemia. Eur. $J$. Cancer Care (Engl). 13(3), 279287 (2004).

19. Sivakumaran M, Qureshi H, Chapman CS, Chylous effusions in CLL. Leuk. Lymphoma 18, 365366 (1995).

20. Elliott MA, Letendre L, Li Cy, et al. Chronic lymphocytic leukaemia with symptomatic diffuse central nervous system infiltration responding to therapy with systemic fludarabine. $B r . J$. Haemathology $\quad \mathbf{1 0 4}, \quad 689-694$ (1999).

21. Montserrat E, Moreno C, Chronic lymphocytic leukaemia: a short overview. Ann. Oncol. 19(7), 320325 (2008).

22. Swerdlow S, Campo E, Harris NL, et al. WHO Classification of Tumours of Haematopoietic and Lymphoid Tisues. (Lyon, IARC, 2008) $\square$ 4th ed. $\square$.

23. Nakamura Naoya, Masafumi Abe, Richter syndrome in B-cell chronic lymphocytic leukemia. Pathology International 53 (4), 195-203 (2003).

24. Mao Z, Leticia Q-M, Mark R, et al. IgVH mutational status and clonality analysis of Richter's transformation: diffuse large B-cell lymphoma and Hodgkin lymphoma in association with Bcell chronic lymphocytic leukemia 
Chronic Lymphocytic Leukemia (CLL)/Small Lymphocytic Lymphoma (SLL) - all the aspects

(B-CLL) represent 2 different pathways of disease evolution. Am. J. Surg. Path. 32, 1605-1614 (2007).

25. Hisada M, Biggar RJ, Greene MH, Fraumeni JF Jr, Travis LB. Solid tumors after chronic lymphocytic leukemia. Blood 98(6), 1979-1981 (2001).

26. Dicker F, Schnittger S, Haferlach $\mathrm{T}$, et al. Immunostimulatory oligonucleotide-induced metaphase cytogenetics detect chromosomal aberrations in $80 \%$ of CLL patients: A study of 132 CLL cases with correlation to FISH, IgVH status, and CD38 expression. Blood 108, 3152-3160 (2006).

27. Baliakas $\mathrm{P}$, Iskas $\mathrm{M}$, Gardiner A, et al. Chromosomal translocations and karyotype complexity in chronic lymphocytic leukemia: a systematic reappraisal of classic cytogenetic data. Am. J. Hematol. 89, 249-255 (2014).

28. Woyach JA, Ruppert AS, Lozanski $\mathrm{G}$, et al. Association of disease progression on ibrutinib therapy with the acquisition of resistance mutations: A single-center experience of 267 patients. J. Clin. Oncol. 32 (15), Abstract 7010 (2014).

29. Thompson PA, Wierda WG, Ferrajoli A, et al. Complex
Karyotype, Rather Than Del(17p), Is Associated with Inferior Outcomes in Relapsed or Refractory CLL Patients Treated with Ibrutinib-Based Regimens. Blood 124, Abstract 229 (2014).

30. Dick FR, Maca RD, The lymph node in chronic lymphocytic lymphoma leukemia. Cancer 41, 283-292 (1978).

31. Jahic A, Iljazovic E, ArnautovicCustovic A, et al. Prognostic significance of bone-marrow pattern and immunophenotypic score in B-chronic lymphocytic leukemia at diagnosis. Med Arh. 65(3), 132-136 (2011).

32. Rai KR, Sawitsky A, Cronkite EP, et al. Clinical staging of chronic lymphocytic leukemia. Blood 46, 219-234 (1975).

33. Binet J, Auquier A, Dighiero G, et al. A new prognostic classification of chronic lymphocytic leukemia derived from a multivariate survival analysis. Cancer 48, 198206 (1981).

34. Krober A, Bloehdorn J, Hafner S, et al. Additional genetic high-risk features such as 11q deletion, 17p deletion, and V3-21 usage characterize discordance of ZAP70 and $\mathrm{VH}$ mutation status in chronic lymphocytic leukemia. $J$. Clin. Oncol. 24, 969-975 (2006). 
Chronic Lymphocytic Leukemia (CLL)/Small Lymphocytic Lymphoma (SLL) - all the aspects

35. Krober A, Seiler T, Benner A, et al. $\mathrm{V}(\mathrm{H})$ mutation status, CD38 expression level, genomic aberrations, and survival in chronic lymphocytic leukemia. Blood 100, 1410-1416 (2002).

36. Oscier D, Wade R, Davis Z, et al. Prognostic factors identified three risk groups in the LRF CLL4 trial, independent of treatment allocation. Haematologica 95, 1705-1712 (2010).

37. Oscier DG, Gardiner AC, Mould SJ, et al. Multivariate analysis of prognostic factors in CLL: clinical stage, IGVH gene mutational status, and loss or mutation of the p53 gene are independent prognostic factors. Blood $\mathbf{1 0 0}$, 1177-1184 (2002).

38. Damle RN, Wasil T, Fais F, et al. Ig $\mathrm{V}$ gene mutation status and CD38 expression as novel prognostic indicators in chronic lymphocytic leukemia. Blood 94, 1840-1847 (1999).

39. Del Poeta G, Maurillo L, Venditti $\mathrm{A}$, et al. Clinical significance of CD38 expression in chronic lymphocytic leukemia. Blood 98, 2633-2639 (2001).

40. Ibrahim S, Keating M, Do KA, et al. $\mathrm{CD} 38$ expression as an important prognostic factor in Bcell chronic lymphocytic leukemia. Blood 98, 181-186 (2001).

41. Gentile M, Mauro FR, Calabrese $\mathrm{E}$, et al. The prognostic value of CD38 expression in chronic lymphocytic leukaemia patients studied prospectively at diagnosis: a single institute experience. $B r . J$. Haematol. 130, 549-557 (2005).

42. Crespo M, Bosch F, Villamor N, et al. ZAP-70 expression as a surrogate for immunoglobulinvariable-region mutations in chronic lymphocytic leukemia. $N$. Engl. J. Med. 348, 1764-1775 (2003).

43. Orchard JA, Ibbotson RE, Davis $\mathrm{Z}$, et al. ZAP-70 expression and prognosis in chronic lymphocytic leukaemia. Lancet 363:105-111 (2004).

44. Del Principe MI, Del Poeta G, Buccisano $\mathrm{F}$, et al. Clinical significance of ZAP-70 protein expression in B-cell chronic lymphocytic leukemia. Blood 108, 853-861 (2006).

45. Tam CS, O'Brien S, Wierda W, et al. Long-term results of the fludarabine, cyclophosphamide, and rituximab regimen as initial therapy of chronic lymphocytic leukemia. Blood 112, 975-980 (2008).

46. Tsimberidou AM, Tam C, Wierda $\mathrm{W}$, et al. Beta-2 microglobulin 
Chronic Lymphocytic Leukemia (CLL)/Small Lymphocytic Lymphoma (SLL) - all the aspects

$(\mathrm{B} 2 \mathrm{M})$ is an independent prognostic factor for clinical outcomes in patients with CLL treated with frontline fludarabine, cyclophosphamide, and rituximab (FCR) regardless of age, creatinine clearance $(\mathrm{CrCl})$ [abstract]. J. Clin.

Oncol. 25, Abstract 7034 (2007).

47. Wierda WG, O'Brien S, Wang X, et al. Characteristics associated with important clinical end points in patients with chronic lymphocytic leukemia at initial treatment. J. Clin. Oncol. 27,16371643 (2009).

48. Dohner H, Stilgenbauer S, Benner $\mathrm{A}$, et al. Genomic aberrations and survival in chronic lymphocytic leukemia. N. Engl. J. Med. 343, 1910-1916 (2000).

49. Austen B, Skowronska A, Baker $\mathrm{C}$, et al. Mutation status of the residual ATM allele is an important determinant of the cellular response to chemotherapy and survival in patients with chronic lymphocytic leukemia containing an 11q deletion. J. Clin. Oncol. 25, 5448-5457 (2007).

50. Tsimberidou AM, Tam C, Abruzzo LV, et al. Chemoimmunotherapy may overcome the adverse prognostic significance of $11 \mathrm{q}$ deletion in previously untreated patients with chronic lymphocytic leukemia. Cancer 115(2), 373-380 (2009).

51. Zenz T, Eichhorst B, Busch R, et al. TP53 Mutation and Survival in Chronic Lymphocytic Leukemia. J. Clin. Oncol. 28, 4473-4479 (2010).

52. Stilgenbauer S, Schnaiter A, Paschka $\mathrm{P}$, et al. Gene mutations and treatment outcome in chronic lymphocytic leukemia: results from the CLL8 trial. Blood 123, 3247-3254 (2014).

53. Gonzalez D, Martinez P, Wade R, et al. Mutational status of the TP53 gene as a predictor of response and survival in patients with chronic lymphocytic leukemia: results from the LRF CLL4 trial. J. Clin. Oncol. 29, 2223-2229 (2011).

54. Fabbri G, Rasi S, Rossi D, et al. Analysis of the chronic lymphocytic leukemia coding genome: role of NOTCH1 mutational activation. J. Exp. Med. 208, 1389-1401 (2011).

55. Puente XS, Pinyol M, Quesada V, et al. Whole-genome sequencing identifies recurrent mutations in chronic lymphocytic leukaemia. Nature 475, 101-105 (2011).

56. Wang L, Lawrence MS, Wan Y, et al. SF3B1 and other novel cancer genes in chronic lymphocytic 
Chronic Lymphocytic Leukemia (CLL)/Small Lymphocytic Lymphoma (SLL) - all the aspects

leukemia. N. Engl. J. Med. 365, 497-2506 (2011).

57. Quesada V, Conde L, Villamor N, et al. Exome sequencing identifies recurrent mutations of the splicing factor SF3B1 gene in chronic lymphocytic leukemia. Nat. Genet. 44, 47-52 (2012).

58. Rossi D, Fangazio M, Rasi S, et al. Disruption of BIRC3 associates with fludarabine chemorefractoriness in TP53 wildtype chronic lymphocytic leukemia. Blood 119, 2854-2862 (2012).

59. Oscier DG, Rose-Zerilli MJ, Winkelmann $\mathrm{N}$, et al. The clinical significance of NOTCH1 and SF3B1 mutations in the UK LRF CLL466, trial. Blood 121, 468-475 (2013).
60. Rossi D, Rasi S, Spina V, et al. Different impact of NOTCH1 and SF3B1 mutations on the risk of chronic lymphocytic leukemia transformation to Richter syndrome. Br. J. Haematol. 158, 426-429 (2012).

61. Villamor N, Conde L, MartinezTrillos A, et al. NOTCH1 mutations identify a genetic subgroup of chronic lymphocytic leukemia patients with high risk of transformation and poor outcome. Leukemia 27, 1100-1106 (2013).

62. Kovacs G, Boettcher S, Bahlo J, et al. Value of Minimal Residual Disease (MRD) Negative Status at Response Evaluation in Chronic Lymphocytic Leukemia (CLL): Combined Analysis of Two Phase III Studies of the German CLL Study Group (GCLLSG). Blood 124, Abstract 23 (2014). 